\title{
Traffic pollution exposure is associated with altered brain connectivity in school children
}

Jesus Pujol, MD ${ }^{\mathrm{a}, \mathrm{b}}$; Gerard. Martínez-Vilavella, MSc ${ }^{\mathrm{a}}$; Dídac Macià, MSc ${ }^{\mathrm{a}}$; Raquel Fenoll, MSc ${ }^{\text {a }}$; Mar Alvarez-Pedrerol, $\mathrm{PhD}^{\mathrm{c}, \mathrm{d}, \mathrm{e}}$; Ioar Rivas, MSc ${ }^{\mathrm{c}, \mathrm{de}, \mathrm{f}} ;$ Joan Forns, $\mathrm{Ph}^{\mathrm{c}, \mathrm{de}}$; Laura Blanco-Hinojo, MSc ${ }^{\mathrm{a}}$; Jaume Capellades, $\mathrm{MD}^{\mathrm{g}}$; Xavier Querol, $\mathrm{PhD}^{\mathrm{f}}$; Joan Deus, $\mathrm{PhD}^{\mathrm{a}, \mathrm{h}, \mathrm{i}}$; Jordi Sunyer, MD ${ }^{\mathrm{c}, \mathrm{d}, \mathrm{e}, \mathrm{j}}$

${ }^{a}$ MRI Research Unit, Hospital del Mar, Barcelona, Spain

${ }^{\mathrm{b}}$ Centro Investigación Biomédica en Red de Salud Mental, CIBERSAM G21, Barcelona, Spain ${ }^{\mathrm{c} C e n t r e ~ f o r ~ R e s e a r c h ~ i n ~ E n v i r o n m e n t a l ~ E p i d e m i o l o g y ~(C R E A L), ~ B a r c e l o n a, ~ C a t a l o n i a, ~ S p a i n ~}$ ${ }^{\mathrm{d}}$ Pompeu Fabra University, Barcelona, Catalonia, Spain

${ }^{\mathrm{e}}$ Ciber on Epidemiology and Public Health (CIBERESP), Spain.

fInstitute of Environmental Assessment and Water Research (IDAEA-CSIC), Barcelona,

Catalonia, Spain

${ }^{\mathrm{g}}$ Radiology Department, Hospital del Mar, Barcelona, Spain

${ }^{\mathrm{h}}$ Department of Clinical and Health Psychology, Autonomous University of Barcelona, Spain.

i Instituto Universitario de Neurorrehabilitación Guttmann, Badalona, Spain

jIMIM (Hospital del Mar Medical Research Institute), Barcelona, Catalonia, Spain

Corresponding author: Dr. Jesus Pujol. MRI Department, Hospital del Mar, Passeig Marítim 25-29. 08003, Barcelona, Spain. Email: 21404jpn@ comb.cat Telephone: +34932212180 Fax: +34932212181 


\begin{abstract}
Children are more vulnerable to the effects of environmental elements due to their active developmental processes. Exposure to urban air pollution has been associated with poorer cognitive performance, which is thought to be a result of direct interference with brain maturation. We aimed to assess the extent of such potential effects of urban pollution on child brain maturation using general indicators of vehicle exhaust measured in the school environment and a comprehensive imaging evaluation. A group of 263 children, aged 8 to 12 years, underwent MRI to quantify regional brain volumes, tissue composition, myelination, cortical thickness, neural tract architecture, membrane metabolites, functional connectivity in major neural networks and activation / deactivation dynamics during a sensory task. A combined measurement of elemental carbon and NO2 was used as a putative marker of vehicle exhaust. Air pollution exposure was associated with brain changes of a functional nature, with no evident effect on brain anatomy, structure or membrane metabolites. Specifically, a higher content of pollutants was associated with lower functional integration and segregation in key brain networks relevant to both inner mental processes (the default mode network) and stimulus-driven mental operations. Age and performance (motor response speed) both showed the opposite effect to that of pollution, thus indicating that higher exposure is associated with slower brain maturation. In conclusion, urban air pollution appears to adversely affect brain maturation in a critical age with changes specifically concerning the functional domain.
\end{abstract}

Keywords: brain development, air pollution, functional MRI, functional connectivity. 


\section{Introduction}

Common to living beings, the brain development cycle is characterized by primary growth and subsequent maturation. Maturation changes implicate structure and function with anatomical shaping, progressive myelination of neural tracks and fine-tuning of functional brain networks (Toga et al., 2006; Pujol et al., 2006; Menon, 2013). The highest-order events procure the integration of brain areas into functional systems and the segregation of distinct but interconnected large-scale networks (Uddin et al., 2010; Vogel et al., 2010: Dwye et al., 2014; Di Martino et al., 2014).

Developing children are at risk due to the potentially hazardous effects of environmental factors (Paus, 2010). Long-term exposure to traffic-related air pollution has been associated with alterations in children's cognition (Suglia et al., 2008; Wang et al., 2009; Perera et al., 2009). We have recently identified a significant association between general markers of road traffic pollution and slower cognitive growth in a large group of children (Sunyer et al., 2015).

Epidemiological studies, therefore, indicate that high levels of urban air pollution may be dangerous to children, as they presumably interfere with brain maturation processes. This hypothesis is largely supported by a set of studies in both animals and humans showing significant associations of pollutant exposure with inflammatory and degenerative brain pathology (Block and Calderón-Garcidueñas, 2009; CalderónGarcidueñas, 2012). However, such an interference effect on brain development has not been thoroughly investigated. We aimed to assess the extent of potential repercussions of traffic pollution exposure on child brain maturation using a variety of imaging measurements ranging from basic anatomy to high-order functional integration. A group 
of 263 children, aged 8 to 12 years, recruited from a large study assessing the impact of long-term exposure to urban pollution in Barcelona city school environments (Sunyer et al., 2015) completed the protocol.

Our hypothesis was that the potential brain effects of air pollution will be more evident on the more detectable anatomical and functional maturation processes. Whereas developmental changes in gray matter volume are less evident in this age period, active myelination implicates increases of relative white matter volumes, elevated choline compounds and water diffusion changes within white matter tracts (Toga et al., 2006; Blüml et al., 2013; Yoshida et al., 2013). At the functional domain, preadolescence is critical to the optimal assembling of large-scale functional networks (Menon, 2013). Accordingly, the imaging protocol included a high resolution 3D anatomical acquisition to measure regional volumes, brain tissue composition, myelination levels and cortical thickness. Diffusion tensor imaging (DTI) measurements of fractional anisotropy served to explore white matter tract architecture. In vivo spectroscopy was used to grossly estimate precursors of membrane components in white matter. Finally, functional MRI was used to test the integrity of relevant networks using both resting-state functional connectivity and a task activation/deactivation paradigm.

Selected cognitive assessment was also conducted to determine to what extent potential repercussions were also detectable on children's performance in the current study sample. 


\section{Methods}

\subsection{Participant selection.}

This study was developed in the context of the BREATHE project (The European Commission: FP7-ERC-2010-AdG, ID 268479). The general project design is fully described in Sunyer et al. (2015). A total of 1,564 families, from 39 schools in the city of Barcelona, were invited to participate in the MRI study via post, email or telephone, and 810 of them gave an initial positive response. The study sample was consecutively recruited from this group with the aim of including children from all participating schools. Parents of 491 children were directly contacted. Consent to participate was finally not obtained in 165 cases, 27 children were lost before the assessment and 21 children were not eligible because of dental braces. The finally selected study group included 278 cases. A total of 263 children completed the imaging protocol (mean age of 9.7 years, SD 0.9 and range, 8.0 to 12.1 years). Table 1 reports the characteristics of these participants. Additional cases were excluded on the basis of image quality criteria in each specific MRI analysis (see further).

All parents or tutors signed the informed consent form approved by the Research Ethical Committee (No. 2010/41221/I) of the IMIM-Parc de Salut Mar, Barcelona, Spain and the FP7-ERC-2010-AdG Ethics Review Committee (268479-22022011).

\subsection{Pollutant exposure.}

Each school was measured twice during one-week periods separated by 6 months, in the warm (year 2012) and cold (year 2012/2013) seasons. Indoor air in a single classroom and outdoor air in the playground were measured simultaneously. Pollutants were 
measured during class-time using methods previously described (Sunyer et al., 2015; Amato et al., 2014; Rivas et al., 2014).

Elemental carbon was measured during $8 \mathrm{~h}(09: 00$ to 17:00 h) in particulate matter with an aerodynamic diameter $<2.5 \mu \mathrm{m}(\mathrm{PM} 2.5)$ collected on filters with High-Volume samplers (MCV SA, Spain) using a Thermo Optical Transmission method (Sunset Laboratories Inc.). We carefully followed the EUSAAR-2 protocol, TOT Sunset Laboratories measurements, with a detection limit of $0.1 \mu \mathrm{g} / \mathrm{m} 3$ and an uncertainty of $\pm 5 \%$. The air cleaning effect of High-Volume samplers may underestimate absolute measurements of elemental carbon in poorly ventilated indoors. In our study, however, elemental carbon penetration was almost 1(indoor/outdoor ratio 94.1\% [95\% CI 85.7\%102.4\%]), which suggests a permanent ventilation of the measured classrooms. Elemental carbon was additionally measured in each classroom using the MicroAeth AE51 (AethLabs). The correlation between elemental carbon measured through HighVolume samplers and with the aethelomter was 0.95, supporting that High-Volume sampler measurements may be adequate estimations of classroom elemental carbon.

Nitrogen dioxide (NO2) was measured with passive dosimeters (Gradko). The dosimeter was exposed during a period of 96h (4 days) from Monday to Thursday in each school in both the warm and cold campaigns. Weekly data from both seasons were averaged to obtain a single measurement. Prior to the campaigns, we tested Gradko NO2 passive dosimeters in our urban background monitoring station Palau Reial (with relatively low NO2 concentrations) by measuring during 4 days and comparing the results with simultaneous chemiluminescence NO2 online data. The results showed that sampling periods of 4 days were enough for ensuring a good precision. Also, during the 
whole sampling campaign, NO2 was measured each week (from Monday to Thursday) with both the Gradko passive dosimeter and conventional chemiluminiscence analyzers in this reference station. We obtained a correlation of Gradko $=0.85 *$ chemiluminescence $+3.6(\mathrm{R} 2=0.7)$ for a mean of $37 \mu \mathrm{gNO} 2 / \mathrm{m} 3$, and an uncertainty of $\pm 17 \%$.

We operationally selected elemental carbon and NO2 to compute a general traffic pollution indicator given their relation to vehicle exhaust emissions in the city of Barcelona (Amato et al., 2014). Our interest here was not to identify specific neurotoxic agents directly responsible for neuronal damage, but it was merely to use a measurement globally representing exposure to this sort of air contamination. NO2 is highly correlated with elemental carbon in Barcelona and shows the two typical rush hour peaks (Reche et al., 2011). Barcelona has a diesel dominated vehicle fleet (with very high NOx emissions and a high NO2/NOx rate) with a primary NO2 driven daily pattern (Reche et al., 2011). In Barcelona, $80 \%$ of the NOx emissions arise from road traffic (Catalonian Government Emission Inventory, 2011-2015 Air Quality. http://airuse.eu/wp-content/uploads/2012/10/Kick-off_Generalitat.pdf), and even if a fraction of $\mathrm{NO} 2$ is secondary, this is arising in a large proportion from NO emitted from road traffic as well (Grice et al., 2009). We did not use in this study levels of ultrafine particles, which may be more directly related to the potential neural damage, because in high insolation cities such as Barcelona, levels of ultrafine particles are highly influenced by photochemical nucleation usually occurring at midday. This effect prevents a high correlation of ultrafine particle levels with other traffic tracers (Reche et al., 2011; Brines et al., 2015). 
To obtain more representative measurements, elemental carbon and NO2 were adjusted for temporal variability using whole-year data from a background monitoring station in Barcelona (Sunyer el al., 2015). A single traffic-related pollutant indicator was computed using the weighted average of elemental carbon and NO2 [(EC/group median $)+(\mathrm{NO} 2 /$ group median $) / 2)]$ of pooled indoor and outdoor measurements from both cold and warm seasons. All children had been in the school for more than 18 months (and 98\% more than two years) at imaging assessment, which was carried out after the pollution measurement campaigns.

\subsection{Cognitive performance measurements.}

General cognitive assessment included working memory, motor response speed and attention. Working memory was assessed using a computerized version of the N-Back task (Anderson, 2002). Children's correct detections on 2-back and 3-back loads for number items were used. Specifically, the n-back parameter analyzed was "detectability", a measure of detection subtracting the normalized false alarm rate from the hit rate ( $\mathrm{Z}$ hit rate $-\mathrm{Z}$ false alarm rate). A higher detectability indicates more accurate test performance. Attention and speed of motor responses were assessed using the computerized "Attentional Network Test", child version (Child ANT) (Rueda et al., 2004). We used the overall "reaction time" to measure speed of motor responses and reaction time standard deviation to measure trial-to-trial variability. A higher reaction time standard deviation indicates lower executive and general attentional resources (Langner and Eickhoff, 2013). The ANT task also uses a set of cued and congruent/incongruent conditions to measure specific attention features such as “alerting”, “orienting” and “interference". Details for administering both ANT and NBack tasks are fully described in a previous report (Forns et al., 2014). 
Additional contextual assessments. Socio-demographic factors were measured using a neighborhood socioeconomic status vulnerability index (based on level of education, unemployment and occupation at the census tract) (Atlas de Vulnerabilidad Urbana de España, 2012. http://www.fomento.gob.es/... /Atlas Vulnerabilidad Urbana/) according to both school and home address. Distance from home to school was estimated based at the geocoded postal address of each participant and school. Parental education was registered for both parents using a 5-point scale (1 illiterate/ 2 less than/ 3 primary/ 4 secondary/ 5 university). Standard measurements of height and weight were performed to define overweight and obesity (de Onis et al., 2009). Parents completed the Strengths and Difficulties Questionnaire (SDQ) on child behavioral problems (Goodman, 2001). A “difficulties" score ranging from 0 to 40 was generated. Overall school achievement was rated by teachers using a 5-point scale (from the worse $=1$ to the best $=5$ ).

\subsection{MRI and MR spectroscopy acquisition}

A 1.5 Tesla Signa Excite system (General Electric, Milwaukee, WI, USA) equipped with an eight-channel phased-array head coil and single-shot echoplanar imaging (EPI) software was used. The imaging protocol involved an anatomical T1-weighted 3D sequence, diffusion tensor imaging (DTI), MR proton spectroscopy and functional MRI.

High-resolution 3D anatomical images were obtained using an axial T1-weighted three-dimensional fast spoiled gradient inversion recovery-prepared sequence. A total of 134 contiguous slices were acquired with inversion time $400 \mathrm{~ms}$; repetition time 11.9 ms; echo time $4.2 \mathrm{~ms}$; flip angle $15^{\circ}$; field of view $30 \mathrm{~cm}$; 256 x 256 pixel matrix; slice thickness $1.2 \mathrm{~mm}$. 
Diffusion tensor imaging (DTI) was obtained using spin-echo single-shot echo-planar sequences of 25 directions with a B-factor of $1000 \mathrm{~s} / \mathrm{mm} 2$. Twenty-six slices were acquired with repetition time $8300 \mathrm{~ms}$; echo time $94 \mathrm{~ms}$; thickness $5 \mathrm{~mm}$, no gap; pulse angle $90^{\circ}$; field of view $26 \mathrm{~cm}$; $128 \times 128$ acquisition matrix reconstructed into a $256 \mathrm{x}$ 256 matrix.

Magnetic resonance spectroscopy. Proton $(1 \mathrm{H})$ spectroscopy was performed using the fully automated Proton Brain Exam-Single Voxel (PROBE-SV) software package (GE Medical Systems, Milwaukee, WI) and a Stimulated Echo Acquisition Mode (STEAM) pulse sequence with $\mathrm{TR} / \mathrm{TE}=2000 / 30 \mathrm{~ms}$ and 128 signal averages. Total acquisition time was $5 \mathrm{~m} 4 \mathrm{~s}$. The voxel showed a dimension of $23 \times 14 \times 14 \mathrm{~mm}$ and was always placed in the left frontal white matter with the aid of high-resolution 3-D images. Orthogonal projections in the three planes assisted the placement of the voxel. The major axis (23 $\mathrm{mm})$ was aligned along the anterior-posterior direction in the frontal white matter. Care was taken to minimally include gray matter and to place the voxel just above the caudate nucleus between the cingulate cortex and the frontal cortex at the level of the precentral sulcus.

The functional MRI sequences consisted of gradient recalled acquisition in the steady state with repetition time $2000 \mathrm{~ms}$; echo time $50 \mathrm{~ms}$; pulse angle $90^{\circ}$; field of view 24 cm; 64 x 64-pixel matrix; slice thickness $4 \mathrm{~mm}$ (inter-slice gap, $1.5 \mathrm{~mm}$ ). Twenty-two interleaved slices were prescribed parallel to the anterior-posterior commissure line covering the brain. Two fMRI sequences were acquired for each participant including a 6-min continuous resting-state scan generating 180 whole-brain EPI volumes, and a 4- 
min sensory task generating 120 whole-brain EPI volumes. The first four (additional) images in each fMRI run were discarded to allow magnetization to reach equilibrium.

During the resting-state functional MRI, children were instructed to relax, stay awake and lie still without moving, while keeping their eyes closed throughout. The taskactivation paradigm involved an $\mathrm{ABABABAB}$ block design alternating four 30-s periods of rest (visual fixation to a cross) with four 30-s periods of visual-auditory stimulation delivered using MRI compatible goggles and headphones (VisuaStim Digital, Resonance Technology, USA). Subjects were passively confronted with a set of facial images expressing happiness and with music showing a rapid tempo (Beethoven Symphony No. 6 "Pastorale“).

\subsection{Image/spectra processing}

\subsubsection{D anatomical images}

All the anatomical images were visually inspected before analysis by a trained operator to detect any motion effect. A total of 10 children were discarded as a result of poor quality images and thus the final sample for the 3D anatomical analysis included 253 children. Anatomical 3D data were processed in two separate analyses assessing different anatomical characteristics:

Gray and white matter tissue concentration and volume at a voxel level was measured using Statistical Parametric Mapping (SPM8) (http://www.fil.ion.ucl.ac.uk/spm, Wellcome Department of Cognitive Neurology, London, UK, 2008). SPM voxel-based morphometry (VBM) algorithms with DARTEL registration were used with the following processing steps: segmentation of anatomical images into gray and white 
matter tissue probability maps in their native space; estimation of the deformations that best align the images together by iteratively registering the segmented images with their average; finally, generating spatially normalized and smoothed segmentations $(5 \times 5 \times 5$ FWHM) using the deformations estimated in the previous step. The analyses were performed with scaling by Jacobian determinants (estimates of volume change during the normalization) to consider tissue volume and without Jacobian scaling to assess the relative concentration of gray matter and white matter. Normalized images were finally transformed to the standard SPM template, re-sliced to $1.5 \mathrm{~mm}$ resolution in Montreal Neurological Institute (MNI) space.

Cortical thickness measurements across the whole cortex were obtained using FreeSurfer tools (http://surfer.nmr.mgh.harvard.edu/). Processing steps included removal of non-brain tissue, segmentation of the subcortical white matter and deep gray matter volumetric structures, tessellation of the gray and white matter boundary, registration to a spherical atlas which is based on individual cortical folding patterns to match cortical geometry across subjects and creation of a variety of surface based data. Cortical thickness is calculated as the closest distance from the gray/white boundary to the gray/CSF boundary at each vertex on the tessellated surface (Fischl et al., 1999).

\subsubsection{Diffusion tensor imaging (DTI)}

DTI was processed using Functional MRI of the Brain (FMRIB) Software Library 5.0 (FSL), developed by the Analysis Group at the Oxford Centre for FMRIB (Smith et al., 2004). Diffusion-weighted images were corrected for motion and eddy current distortions ("Eddy Current Correction" option in the FMRIB Diffusion Toolbox [FDT] version 2.0 in FSL), and a whole-brain mask was applied using the FSL Brain 
Extracting Tool. A further rigorous image quality control was carried out to identify potential residual effects of head motion, which involved the visual inspection of each DTI slice for all 25 DTI volumes in all participants. Volumes with slices with signal loss (greater than $\sim 10 \%$ ) or residual artifacts were identified by an expert researcher. DTI full examinations showing one or more degraded images in more than 5 volumes were discarded. A total of 76 children were removed from the DTI analysis on the basis of this criterion (in addition to 10 cases showing gross image degradation). The final DTI sample involved 177 children showing a mean \pm SD of $23.5(94 \%) \pm 1.9$ optimalquality volumes. Subsequently, we estimated fractional anisotropy (FA) maps using FDT in FSL after local fitting of the diffusion tensor model at each voxel ("dtifit"). Next, diffusion data were processed using Tract- Based Spatial Statistics (Smith et al., 2006). Each FA data set was re-sliced to a $1 \mathrm{~mm} \times 1 \mathrm{~mm} \times 1 \mathrm{~mm}$ anatomical resolution and normalized to standard MNI space via the FMRIB58_FA template using the FMRIB's Non-linear Registration Tool.

\subsubsection{Magnetic resonance spectroscopy}

Metabolite relative measurements were performed on the Advantage Windows, v. 4.2, workstation using the PROBE-SV software package, which includes automatic processing of the raw data that permits immediate display and evaluation of spectra. Measurements of choline-containing compounds (choline-to-total creatine ratio) were used to test the association of air pollution with membrane precursors in white matter (Blüml et al., 2013). N-acetylaspartate (NAA) spectra width and the ratio creatine-tonoise (root-mean-square 'RMS' noise) were used to assess spectra quality. The quality criteria to be retained for statistical analyses were NAA linewidth (full width at half maximum of peak) of $0.09 \mathrm{ppm}$ or less and creatine RMS noise of 8 or greater. A total 
of 34 participants were excluded as a result of poor spectrum quality. The final sample for the spectroscopy analysis included 229 children.

\subsubsection{Functional MRI}

Resting-state and task functional MRI preprocessing was carried out using SPM8 and involved motion correction, spatial normalization and smoothing using a Gaussian filter (full-width half-maximum, $8 \mathrm{~mm}$ ). Data were normalized to the standard SPM-EPI template and re-sliced to $2 \mathrm{~mm}$ isotropic resolution in MNI space.

The following procedures were adopted to control for potential head motion effects: (i) Conventional SPM time-series alignment to the first image volume in each subject. (ii) Exclusion of 24 children in the resting-state analysis and 39 children in the taskactivation analysis with large head motion. That is, outliers (and extremes) with regard to mean inter-frame motion were excluded using conventional boxplot criteria (cases beyond the quartile Q3 by one-and-a-half Q3-Q1 interquartile range [SPSS 15.0; SPSS Inc., Chicago IL]). The finally analyzed sample therefore included 239 children with valid resting-state and 224 children with valid task assessment. (iii) Both motion-related regressors (a total of 6 realignment parameters, including 3 translation and 3 rotation first-order derivatives) and estimates of global brain signal fluctuations were included as confounding variables in first-level (single-subject) analyses. (iv) Within-subject, censoring-based MRI signal artifact removal (scrubbing) (Power et al., 2014) was used to discard motion-affected volumes. For each subject, inter-frame motion measurements (Pujol et al., 2014b) served as an index of data quality to flag volumes of suspect quality across the run. At points with inter-frame motion $>0.2 \mathrm{~mm}$, that corresponding volume, the immediately preceding and the succeeding two volumes were discarded. Using this 
procedure, a mean \pm SD of $11.2(6.2 \%) \pm 13.8$ volumes out of 180 fMRI resting-state sequence volumes and $14.9(12.4 \%) \pm 15.8$ volumes out of the 120 fMRI task volumes were removed. (v) Potential motion effects were further removed using a summary measurement for each participant (mean inter-frame motion across the fMRI run) as a regressor in the second-level (group) analyses in SPM (Pujol et al., 2014b).

Resting-state fMRI. Four functional connectivity MRI maps were generated using coordinates taken from a classical study (Fox et al., 2005), converted to MNI in mm and located at the medial frontal cortex $[x=1, y=54, z=26]$, posterior cingulate cortex $[x=-2$, $\mathrm{y}=-38, \mathrm{z}=38]$, dorsal frontal cortex $[\mathrm{x}=28, \mathrm{y}=-10, \mathrm{z}=58]$ and supplementary motor area $[\mathrm{x}=-2, \mathrm{y}=-2, \mathrm{z}=55]$.

The maps obtained using the medial frontal cortex and the posterior cingulate cortex regions typically include all the elements of the Default Mode Network (DMN), which is highly active in inner mental processes and is negatively correlated (anticorrelated) with networks participating in attention-demanding tasks (Kelly et al., 2008). On the other hand, maps from the frontal cortex and supplementary motor area regions both capture networks commonly participating in attention-demanding tasks. As classically described, the dynamic relationship between the DMN and the anticorrelated taskrelated networks may reflect the largest-scale functional organization in the brain (Kelly et al., 2008; Fox et al., 2005). Interestingly, this network interaction maturates significantly in the age period targeted in the present study (Sherman et al., 2014).

Functional connectivity maps were generated using procedures detailed in previous reports (Harrison et al., 2013; Pujol et al., 2014a). For each location, the seed region 
was defined as a $3.5 \mathrm{~mm}$ radial sphere (sampling 25 voxels in $2 \mathrm{~mm}$ isotropic space). This was performed using MarsBaR region of interest (ROI) toolbox in MNI stereotaxic space (Brett et al., 2003). Signals of interest were then extracted for each seed region respectively by calculating the mean ROI value at each time point across the timeseries. To generate the seed maps, the signal time course of a selected seed region was used as a regressor to be correlated with the signal time course of every voxel in the brain in order to generate first-level (single-subject) voxel-wise statistical parametric maps (contrast images). The maps were estimated for each seed separately. A high-pass filter set at $128 \mathrm{~s}$ was used to remove low frequency drifts below $\sim .008 \mathrm{~Hz}$. In addition, we derived estimates of withe matter, CSF and global brain signal fluctuations (using standard masks in MNI space from SPM) to include in the regression analyses as nuisance variables.

Task-activation fMRI. Functional interaction between brain systems may also be inferred using fMRI task paradigms by assessing the segregation between activated and deactivated areas during stimulation (Harrison et al., 2008; Pujol et al., 2012). Deactivated areas during our sensory task include part of the DMN, which are generally anticorrelated with networks participating in attention-demanding tasks (Kelly et al., 2008; Chai et al., 2014), although the deactivation pattern is not limited to the DMN. In the task analysis, single-subject (first-level) SPM contrast images were estimated for activations (stimulation condition > rest) and deactivations (rest $>$ stimulation condition). For these analyses, the fMRI signal response at each voxel was modeled using the SPM canonical hemodynamic response function. 
Before the statistical analysis, all the processed images (anatomical 3D, DTI, and functional MRI) were visually inspected to detect processing-induced artifacts and verify the accuracy of anatomical segmentation and FreeSurfer surface reconstructions.

\subsection{Statistical analysis.}

Data was treated as quantitative variables and the analyses involved cross-correlations using SPM. After individual preprocessing of each imaging exam, separate second-level analyses were carried out to map voxel-wise the correlation across-subjects between individual brain images and individual age and pollutant measurements (obtained in the school of each participant). The correlations with pollutant measurements were performed both with and without adjusting by age and sex. The set of individual brain images included whole-brain estimates of regional white matter and gray matter volume and concentration, cortical thickness, DTI fractional anisotropy, resting-state functional connectivity and task-activation/ deactivation. The association with choline-to-creatine metabolite ratio was obtained using simple bivariate Pearson correlation. Total creatine was examined first for an association with traffic pollution. We found no association. As mentioned, the number of participants finally included in each analysis after exclusions on the basis of image quality criteria was: anatomic T1-weighted images, 253; DTI, 177; MR spectroscopy, 229; resting-state fMRI, 239; and sensory task fMRI 224. Finally, three additional children with outlier pollutant measurements were excluded from the correlation between brain measurements and air pollution.

Results were considered significant with clusters of $1.032 \mathrm{ml}$ (e.g., 129 voxels with a resolution of $2 \times 2 \times 2 \mathrm{~mm}$ ) at a height threshold of $\mathrm{p}<0.005$, which satisfied the familywise error $(\mathrm{FWE})$ rate correction of $\mathrm{P}_{\mathrm{FWE}}<0.05$ according to Monte Carlo simulations 
(Pujol et al., 2014c). Resting-state fMRI data were additionally adjusted for multiple testing (four functional connectivity maps) using Bonferroni (significant cluster size $\geq$ $1.4 \mathrm{ml})$. Maps in figures are displayed at $\mathrm{t}>2.3$. 


\section{Results}

Traffic-related pollution at the children's schools was assessed using the weighted average of two reliable vehicle exhaust indicators, namely particulate elemental carbon and NO2 (Methods). Table 1 reports ambient air concentrations of the measured pollutants. According to these data, air pollution levels at school in the city of Barcelona may be considered moderate-to-high when compared with other areas (Eeftens et al., 2012; Cyrys et al., 2012).

This summary pollution index was then whole-brain correlated with several MRI maps characterizing brain maturation. No significant association was identified between air pollution and any anatomical, structural or metabolic brain measurement. By contrast, the functional imaging analysis showed consistent results.

Our functional approach involved the generation of connectivity maps representative of key neural networks using coordinates taken from previous works (see Methods). The map obtained using the medial frontal cortex (seed) region of interest produced the most illustrative results. We found that traffic-related air pollution was significantly associated with weaker functional connectivity between regions belonging to the DMN (i.e., between the medial frontal cortex and the angular gyrus bilaterally), indicating lower intra-network integration (Fair et al., 2009). In addition, pollution also was associated with stronger functional connectivity between the (medial frontal cortex) seed region and the frontal operculum at the lateral boundary of the DMN, indicating lower network segregation (Fair et al., 2009) (Figure 1 and Table 2). 
The analysis of the correlation between children's age and functional connectivity in the DMN was useful in establishing the detrimental nature of the results (Figure 2). Indeed, the age effect on functional connectivity in this network was the opposite of the pollutant effect. Age was significantly associated with stronger functional connectivity within the elements of the DMN (integration) and significantly associated with weaker functional connectivity with the bordering network (segregation), as shown in a previous longitudinal study (Sherman et al., 2014). Figure 3 illustrates the opposite effects of age and pollutants using a 3D rendering display.

The analyses based on additional functional connectivity maps mirrored such findings with results in the same direction. The maps included the DMN identified from a seed region located in the posterior cingulate cortex (Table 2) and anticorrelated networks generated from dorsal frontal cortex (Supplementary Figure 1) and supplementary motor area seeds (Supplementary Figure 2).

The potential effects of air pollution on brain function were further tested by mapping the correlation between pollutant measurements and fMRI task activations and deactivations. Air pollutants were significantly associated with lower deactivations (rest $>$ task map) during passive viewing and listening in the supplementary motor area and somatosensory cortex (Figure 4). No significant findings were observed in the task > rest map.

To test whether measured pollution was associated with cognitive performance, we used children's performance in working memory, motor response speed and attention (Methods). The only significant result involved motor speed. Higher pollution predicted 
slower reaction time in 248 participants with complete MRI and behavioral testing (standardized $\beta=0.154 ; p=0.015$ ). A further imaging analysis was performed to correlate children's reaction time with functional connectivity in the DMN map (Figure 5). Interestingly, a faster reaction time was associated with stronger connectivity within the DMN (network integration) and weaker connectivity in the frontal operculum (network segregation).

The effect of potential confounders was tested for each significant finding including age, sex, academic achievement, difficulties scores, obesity, parental education, home and school vulnerability index, distance from home to school and public/non-public school category as covariates. Each potential confounder was both individually entered into the model and combined with other confounders. No single confounder or combination showed a relevant effect. That is, decreases in $\beta$ estimates after the inclusion of confounders in a regression model were very small (mean $\pm \mathrm{SD}, 1.2 \% \pm$ $1.0 \%$ ) with no variables affecting the primary results with $\beta$ reductions greater than 7 $\%$. 


\section{Discussion}

Vehicle exhaust-related air pollution exposure was associated with brain changes of a functional nature, with no evident effect on brain anatomy, structure or membrane metabolism. Children from schools with higher traffic-related pollution showed lower functional integration and segregation in key brain networks. Age and performance (i.e., motor response speed) both showed the opposite effect to that of pollution on brain function, thus indicating that higher exposure is associated with slower brain maturation

The functional findings were highly consistent, as similar effects were observed in different functional networks and the age-sensitive areas notably coincided with the areas showing significant correlation with air pollution. Similarly, the regions identified with the mapping of correlations with motor speed also showed a notable correspondence with the anatomy of findings from both pollutant and age analyses. Nonetheless, despite the evident effect on functional connectivity, the overall brain repercussion may, to some extent, be considered subtle, as changes did not involve any measurement of brain structure. In such a context, one may speculate on the reversibility of the brain damage and the potential effectiveness of actions addressed to reduce pollution. Epidemiological data also support the notion of a subtle repercussion, as large samples are required to demonstrate robust associations (Sunyer et al., 2015).

The effect of air pollution may, however, be more dramatic when the exposure involves early developmental periods. Indeed, Peterson et al. (2015) have provided evidence of brain structural alterations in later childhood associated with prenatal pollutant exposure affecting large areas of the left-hemisphere white matter, and a less severe effect 
associated with postnatal exposures at age 5 years. Also, recent studies have revealed that long-term ambient air pollution exposure may ultimately affect brain tissue volume in older people (Chen et al., 2015; Wilker et al., 2015).

We have used a general marker of vehicle exhaust traffic-related air pollution based on elemental carbon and NO2. Although this indicator reflects the amount of pollution from the traffic source, carbon and NO2 are not necessarily the agents causing the toxic effect on the brain. Traffic pollution contains a variety of elements with greater potential neurotoxicity, such as manganese, aluminum, lead and copper (Amato et al., 2014). We have recently identified the effects of airborne copper pollution, which is road trafficrelated, although a significant proportion also comes from industry, and a third source is the result of railway traffic (submitted). Brain alterations associated with copper were neural system specific and affected the basal ganglia with damage to both structure and function. In contrast, our current study based on a general indicator does not inform on which specific neurotoxicant or a combination thereof may be responsible for the identified effect restricted to functional measurements.

A concern in traffic pollution studies is potential residual confounding by sociodemographic characteristics (e.g., when a relationship exists between proximity to traffic and economically disadvantaged areas). In the city of Barcelona, however, there was a small and inverse relation between air pollution and socioeconomic vulnerability, with higher pollution levels in schools with lower vulnerability (Sunyer et al., 2015). Also, we found no significant associations between school pollution levels and parental education, employment or educational quality. Besides, the associations between air 
pollution and fMRI remained after adjusting for all the potential confounders (see Results), which is opposed to a potential role of these variables.

A general limitation when assessing children with MRI is the potential effect of head movements on image quality, particularly on functional MRI and DTI acquisitions. We considered this issue carefully and adopted several means to rigorously control the effects of motion (Methods). However, it is relevant to emphasize that a rigorous control of potential head motion may introduce spurious changes (e.g., regressing out global brain signal may introduce negative correlations) or even remove changes related to genuine neural activity (Pujol et al., 2014b). Also, a higher MRI signal may be obtained using a higher magnetic field (i.e., 3-Tesla magnets). Although we did have the 3-Tesla option, the present study was developed using a 1.5-Tesla magnet following the recommendations of the FP7-ERC Ethics Review Committee to limit magnetic field strength in children.

\subsection{Conclusion}

Although children's brains may be vulnerable at each developmental stage, preadolescence is notably transcendent in establishing solid bases for large-scale functional network organization. Urban traffic pollution appears to be capable of affecting the normal development of the proto-adult brain and significantly interfering with functional network maturation. 


\section{Acknowledgments}

This work was supported by the European Research Council under the ERC [grant number 268479] - the BREATHE project. The Agency of University and Research Funding Management of the Catalonia Government participated in the context of Research Group SGR2014-1673. We acknowledge Cecilia Persavento, Judit González, Laura Bouso, Mónica López and Pere Figueras their contribution to the field work. We also acknowledge all the families and schools participating in the study.

\section{Conflicts of interest}

The authors declare no conflict of interest. 


\section{References}

Amato F, Rivas I, Viana M, et al. Sources of indoor and outdoor PM2.5 concentrations in primary schools. Sci Total Environ. 2014;490: 757-765.

Anderson P. Assessment and development of executive function (EF) during childhood. Child Neuropsychology. 2002;8: 71-82.

Block ML, Calderón-Garcidueñas L. Air pollution: mechanisms of neuroinflammation and CNS disease. Trends Neurosci. 2009;32: 506-516.

Blüml S, Wisnowski JL, Nelson MD Jr, et al. Metabolic maturation of the human brain from birth through adolescence: insights from in vivo magnetic resonance spectroscopy. Cereb Cortex. 2013;23: 2944-2955.

Brett M, Valabregue R, Poline J. Region of Interest analysis using an SPM toolbox. Neuroimage. 2003:16(Suppl.).

Brines M, Dall'osto M, Beddows DCS, et al. Traffic and nucleation events as main sources of ultrafine particles in high insolation developed world cities. Atmos Chem Phys. 2015;15: 5929-5945.

Chai XJ, Ofen N, Gabrieli JD, Whitfield-Gabrieli S. Selective development of anticorrelated networks in the intrinsic functional organization of the human brain. $\mathbf{J}$ Cogn Neurosci. 2014;26: 501-513.

Calderón-Garcidueñas L. White matter hyperintensities, systemic inflammation, brain growth, and cognitive functions in children exposed to air pollution. J Alzheimer's Dis. 2012;31: 183-191.

Chen JC, Wang X, Wellenius GA, et al. Ambient air pollution and neurotoxicity on brain structure: Evidence from Women's Health Initiative Memory Study. Ann Neurol. 2015;78: 466-476. 
Cyrys J, Eeftens MR, Heinrich J, et al. Variation of NO2 and NOx concentrations between and within 36 European study areas: Results from the ESCAPE study. Atmospheric Environment. 2012;62: 374-390.

de Onis M, Garza C, Onyango AW, Rolland-Cachera MF. WHO development standards for infants and young children. Arch Pediatr. 2009;16: 47-53.

Di Martino A, Fair DA, Kelly C, et al. Unraveling the miswired connectome: a developmental perspective. Neuron. 2014;83: 1335-1353.

Dwyer DB, Harrison BJ, Yücel M, et al. Large-scale brain network dynamics supporting adolescent cognitive control. J Neurosci. 2014;34: 14096-14107.

Eeftens M, Tsai MY, Ampe C, et al. Spatial variation of PM2.5, PM10, PM2.5 absorbance and PMcoarse concentrations between and within 20 European study areas and the relationship with NO2 - Results of the ESCAPE project. Atmospheric Environment. 2012;62: 303-317.

Fair DA, Cohen AL, Power JD, et al. Functional brain networks develop from a "local to distributed" organization. PLoS Comput Biol. 2009;5: e1000381.

Fischl B, Sereno MI, Dale AM. Cortical surface-based analysis. II: Inflation, flattening, and a surface-based coordinate system. Neuroimage. 1999;9: 195-207.

Forns J, Esnaola M, López-Vicente M, et al. The n-back test and the attentional network task as measures of child neuropsychological development in epidemiological studies. Neuropsychology. 2014;28: 519-529.

Fox MD, Snyder AZ, Vincent JL, et al. The human brain is intrinsically organized into dynamic, anticorrelated functional networks. Proc Natl Acad Sci U S A. 2005;102: 9673-9678.

Goodman R. Psychometric properties of the strengths and difficulties questionnaire. J Am Acad Child Adol Psychiatry. 2001;40: 1337-1345. 
Grice S, Stedman J, Kent A et al. Recent trends and projections of primary NO2 emissions in Europe. Atmospheric Environment. 2009;43: 2154-2167.

Harrison BJ, Pujol J, Cardoner N, et al. Brain corticostriatal systems and the major clinical symptom dimensions of obsessive-compulsive disorder. Biol Psychiatry. 2013;73: 321-328.

Harrison BJ, Pujol J, López-Solà M, et al. Consistency and functional specialization in the default mode brain network. Proc Natl Acad Sci U S A. 2008;105: 9781-9786.

Kelly AM, Uddin LQ, Biswal BB, et al. Competition between functional brain networks mediates behavioral variability. Neuroimage. 2008;39: 527-537.

Langner R, Eickhoff SB. Sustaining attention to simple tasks: a meta-analytic review of the neural mechanisms of vigilant attention. Psychol Bull. 2013;139: 870-900.

Menon V. Developmental pathways to functional brain networks: emerging principles. Trends Cogn Sci. 2013;17: 627-640.

Paus T. Population neuroscience: why and how. Hum Brain Mapp. 2010;31: 891-903.

Perera FP, Li Z, Whyatt R, et al. Prenatal airborne polycyclic aromatic hydrocarbon exposure and child IQ at age 5 years. Pediatrics. 2009;124: e195-202.

Peterson BS, Rauh VA, Bansal R, et al. Effects of prenatal exposure to air pollutants (polycyclic aromatic hydrocarbons) on the development of brain white matter, cognition, and behavior in later childhood. JAMA Psychiatry 2015;72 :531-540.

Power JD, Mitra A, Laumann TO, et al. Methods to detect, characterize, and remove motion artifact in resting state fMRI. Neuroimage. 2014;84: 320-341.

Pujol J, Batalla I, Contreras-Rodríguez O, et al. Breakdown in the brain network subserving moral judgment in criminal psychopathy. Soc Cogn Affect Neurosci. 2012;7: 917-923. 
Pujol J, del Hoyo L, Blanco-Hinojo L, et al. Anomalous brain functional connectivity contributing to poor adaptive behavior in Down syndrome. Cortex. 2014a;64C: 148156.

Pujol J, Macià D, Blanco-Hinojo L, et al. Does motion-related brain functional connectivity reflect both artifacts and genuine neural activity? Neuroimage. 2014b;101: 87-95.

Pujol J, Macià D, Garcia-Fontanals A, et al. The contribution of sensory system functional connectivity reduction to clinical pain in fibromyalgia. Pain. 2014c;155: 1492-1503.

Pujol J, Soriano-Mas C, Ortiz H, et al. Myelination of language-related areas in the developing brain. Neurology. 2006;66: 339-343.

Reche C, Querol X, Alastuey A, et al. New considerations for PM, Black Carbon and particle number concentration for air quality monitoring across different European cities. Atmos Chem Phys. 2011;11: 6207-6227.

Rivas I, Viana M, Moreno T, et al. Child exposure to indoor and outdoor air pollutants in schools in Barcelona, Spain. Environ Int. 2014;69: 200-212.

Rueda MR, Fan J, McCandliss BD, et al. Development of attentional networks in childhood. Neuropsychologia. 2004;42: 1029-1040.

Sherman LE, Rudie JD, Pfeifer JH, et al. Development of the default mode and central executive networks across early adolescence: a longitudinal study. Dev Cogn Neurosci. 2014;10: 148-159.

Smith SM, Jenkinson M, Johansen-Berg H, et al. Tract-based spatial statistics: voxelwise analysis of multi-subject diffusion data. Neuroimage. 2006;31: 1487-1505. 
Smith SM, Jenkinson M, Woolrich MW, et al. Advances in functional and structural MR image analysis and implementation as FSL. Neuroimage. 2004; 23 Suppl 1: S208-219.

Suglia SF, Gryparis A, Wright RO, et al. Association of black carbon with cognition among children in a prospective birth cohort study. Am J Epidemiol. 2008;167: 280286.

Sunyer J. Esnaola M, Alvarez-Pedrerol M, et al. Traffic-related air pollution in schools impairs cognitive development in primary school children. PLoS Med. 2015;2: e1001792.

Toga AW, Thompson PM, Sowell ER. Mapping brain maturation. Trends Neurosci. 2006; 29: 148-159.

Uddin LQ, Supekar K, Menon V. Typical and atypical development of functional human brain networks: insights from resting-state FMRI. Front Syst Neurosci. 2010; 4: 21 .

Vogel AC, Power JD, Petersen SE, Schlaggar BL. Development of the brain's functional network architecture. Neuropsychol Rev. 2010;20: 362-375.

Wang S, Zhang J, Zeng X, et al. Association of traffic-related air pollution with children's neurobehavioral functions in Quanzhou, China. Environ Health Perspect. 2009;117: 1612-1618.

Wilker EH, Preis SR, Beiser AS, et al. Long-term exposure to fine particulate matter, residential proximity to major roads and measures of brain structure. Stroke. $2015 ; 46$ :1161-1166.

Yoshida S, Oishi K, Faria AV, Mori S. Diffusion tensor imaging of normal brain development. Pediatr Radiol. 2013;43: 15-27. 
Table 1. Characteristics of the study sample $(n=263)$

Gender

Age, years, mean \pm SD (range)

Overall School Achievement- 5-point scale

Difficulties Score (SDQ), range 0-40

Obesity: normal

Overweight, bmi 85-94

Obesity, bmi $>94$

Mother Education (5-point scale. 5= University)

Father Education (5-point scale. 5= University)

Vulnerability Index ${ }^{\mathrm{a}}$ - Home

Vulnerability Index ${ }^{\mathrm{a}}$ - School

Public/Non-Public School

Task Performance, N-Back

Working memory, 2-back (detectability)

Working memory, 3-back (detectability)

Task Performance, Attentional Network Test

Reaction Time (ms)

Reaction Time Standard Deviation (ms)

Commission Errors (number)

Omission Errors (number)

Alerting (ms)

Orienting (ms)

Interference (ms)

Air pollution measurements ${ }^{\mathrm{b}}$

Outdoor elemental carbon (EC) year average $\left(\mu \mathrm{g} / \mathrm{m}^{3}\right)$

Outdoor NO2 year average $\left(\mu \mathrm{g} / \mathrm{m}^{3}\right)$

Indoor elemental carbon (EC) year average $\left(\mu \mathrm{g} / \mathrm{m}^{3}\right)$

Indoor NO2 year average $\left(\mu \mathrm{g} / \mathrm{m}^{3}\right)$

Overall air pollution indicator (EC+NO2 weighted average)
$48.3 \%$ girls $51.7 \%$ boys

$9.7 \pm 0.9(8.0-12.1)$

$3.7 \pm 1.0(1-5)$

$8.8 \pm 5.3(0-25)$

$71.4 \%$

$18.4 \%$

$10.2 \%$

$4.5 \pm 0.8(1-5)$

$4.4 \pm 0.8(1-5)$

$0.43 \pm 0.21(0.06-0.90)$

$0.43 \pm 0.22(0.13-0.84)$

$43 \%$ vs $57 \%$

$2.5 \pm 1.3(-0.6-3-9)$

$1.5 \pm 1.1(-1.4-3.9)$

$650.6 \pm 119.9(431-1091)$

$222.9 \pm 91.2(77.5-571.6)$

$4.3(3.4 \%) \pm 5.0(0-49)$

$1.6(1.3 \%) \pm 3.9(0-44)$

$53.1 \pm 55.5(-138-270)$

$24.4 \pm 56.8(-204-191)$

$39.4 \pm 35.5(-91-170)$

$1.4 \pm 0.6(0.6-3.99)$

$46.8 \pm 12.0(25.9-84.6)$

$1.2 \pm 0.5(0.4-2.7)$

$29.4 \pm 11.7(11.5-65.6)$

$0.92 \pm 0.30(0.42-1.92)$

${ }^{\mathrm{a}}$ Neighborhood socioeconomic status vulnerability index based on level of education, unemployment, and occupation at the census tract (Atlas de vulnerabilidad urbana de España, 2012). ${ }^{\mathrm{b}}$ After excluding 3 children with outlier measurements. Bmi, body mass index. SDQ, Strengths and Difficulties Questionnaire 
Table 2. Functional MRI results

\section{Correlation with air pollution-}

\section{Medial Frontal Seed Map}

L Lateral Frontal Cortex- positive correlation

L Parietal Cortex- negative correlation

R Parietal Cortex- negative correlation

\section{Dorsal Frontal Seed Map}

L Parietal Cortex- positive correlation

R Lateral Frontal Cortex- negative correlation

$\mathrm{R}$ Insula- negative correlation

\section{Posterior Cingulate Cortex Seed Map}

R Lateral Frontal Cortex- positive correlation

Supplementary Motor Area Seed Map

L Prefrontal Cortex- positive correlation

R Prefrontal Cortex- positive correlation

L Parietal Cortex- positive correlation

R Parietal Cortex- positive correlation

Anterior Cingulate Cortex- negative correlation

\section{Sensory Task}

R Somatosensory Cortex- positive correlation

L Premotor Cortex- positive correlation

\begin{tabular}{cccccc} 
non-adjusted & & \multicolumn{2}{c}{ adj. by age and sex } \\
\hline$m l$ & $x y$ & $t$ & $x y z$ & $t$ \\
\hline
\end{tabular}

$-484616-3.6$

$-524618$

4.1

$2.6 \quad-50-5844 \quad 3.5$

$-48-5644 \quad 3.2$

$1.7 \quad 58-5646 \quad 3.5$

$58-5646 \quad 3.2$

$3.2 \quad-32-6432 \quad 3.6$

$-32-6432 \quad 3.5$

$3.4 \quad 50242 \quad 3.7$

$50242 \quad 3.5$

$1.4 \quad 382-2 \quad 4.3$

$382-2$

4.3

$\begin{array}{lllll}1.5 & 5636-2 & 3.3 & 5636-2 & 3.2\end{array}$

$\begin{array}{llllll}6.1 & -283828 & 4.4 & -283828 & 4.6\end{array}$

$\begin{array}{lllll}2.2 & 224834 & 3.5 & 224834 & 3.6\end{array}$

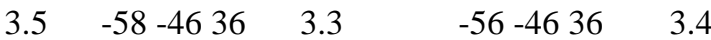

$\begin{array}{lllll}5.2 & 58-4640 & 4.1 & 58-4640 & 3.9\end{array}$

$\begin{array}{lllll}6.2 & -1434-12 & 3.6 & -1436-12 & 3.7\end{array}$

$\begin{array}{lllll}6.3 & 34-4464 & 3.7 & 34-4464 & 3.8 \\ 1.4 & -10060 & 3.7 & -10060 & 3.6\end{array}$

\section{Correlation with age}

\section{Medial Frontal Seed Map}

L Parietal Cortex- positive correlation

Medial Frontal Cortex- positive correlation

Cerebellum- positive correlation

L Lateral Frontal/Insula- negative correlation

R Lateral Frontal/Insula- negative correlation

\begin{tabular}{ccc}
$m l$ & $x y z$ & $t$ \\
\hline 10.7 & $-46-7052$ & 4.9 \\
1.5 & -65248 & 3.3 \\
5.8 & $-22-48-32$ & 4.6 \\
9.7 & -36220 & 4.8 \\
10.4 & 40162 & 3.5
\end{tabular}

\section{Correlation with performance (motor speed)}

\section{Medial Frontal Seed Map}

Medial Frontal Cortex- positive correlation

7.6

$-104626 \quad 3.6$

L Frontal Lateral Cortex- negative correlation

1.6

$-3244-4$

3.9

$\mathrm{x}$ y z, coordinates given in Montreal Neurological Institute (MNI) space. Statistics at corrected threshold $\mathrm{P}_{\mathrm{FWE}}<0.05$ estimated using Monte Carlo simulations. Cluster size in $\mathrm{ml}$. 


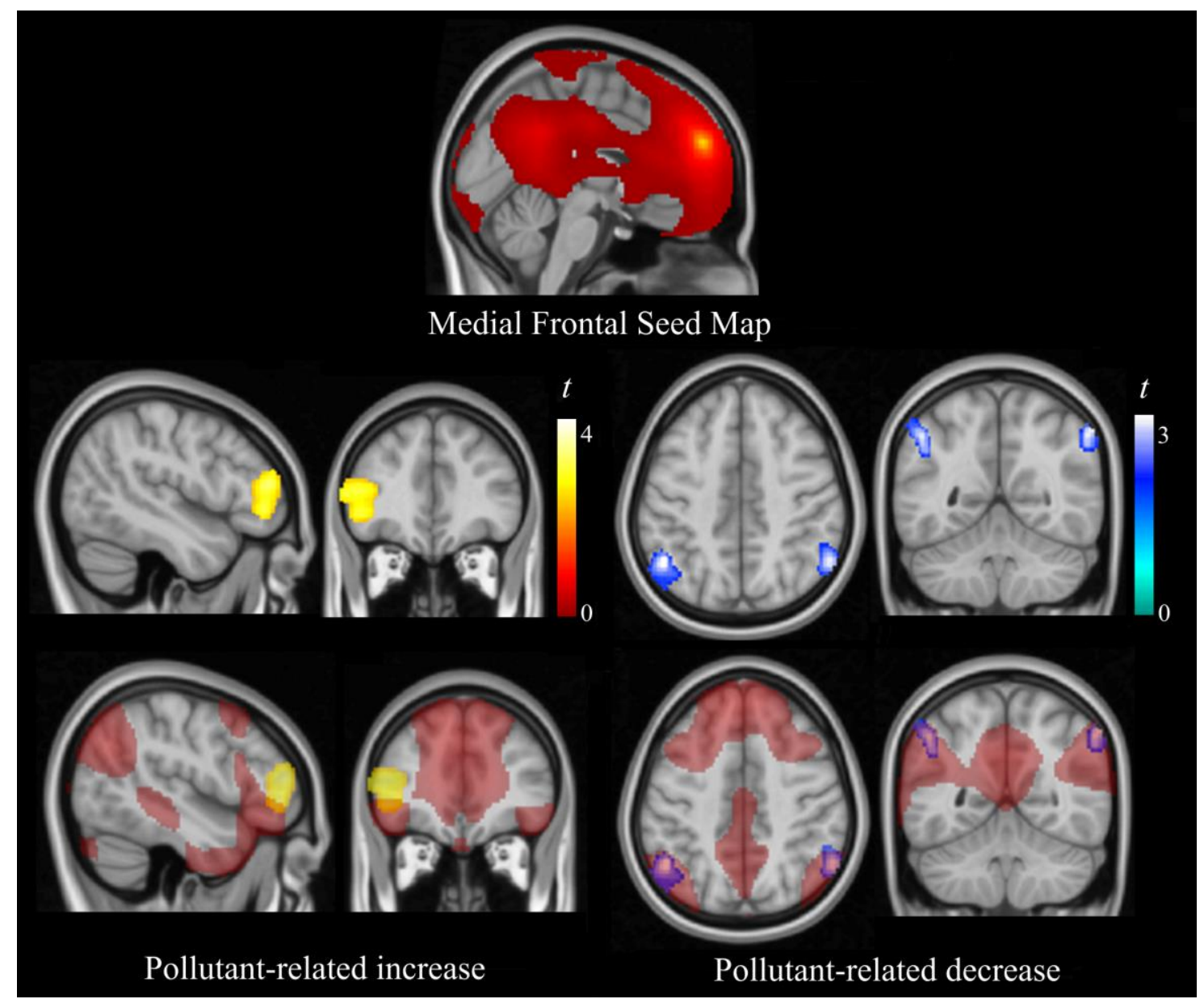

Figure 1. Correlations of urban pollution with functional connectivity in the medial frontal seed map. Higher pollution levels were associated with higher functional connectivity between the medial frontal (seed) region and the lateral frontal cortex (left panels), and lower functional connectivity between the seed and both angular gyri (right panels). The right hemisphere corresponds to the right side of axial and coronal views. 


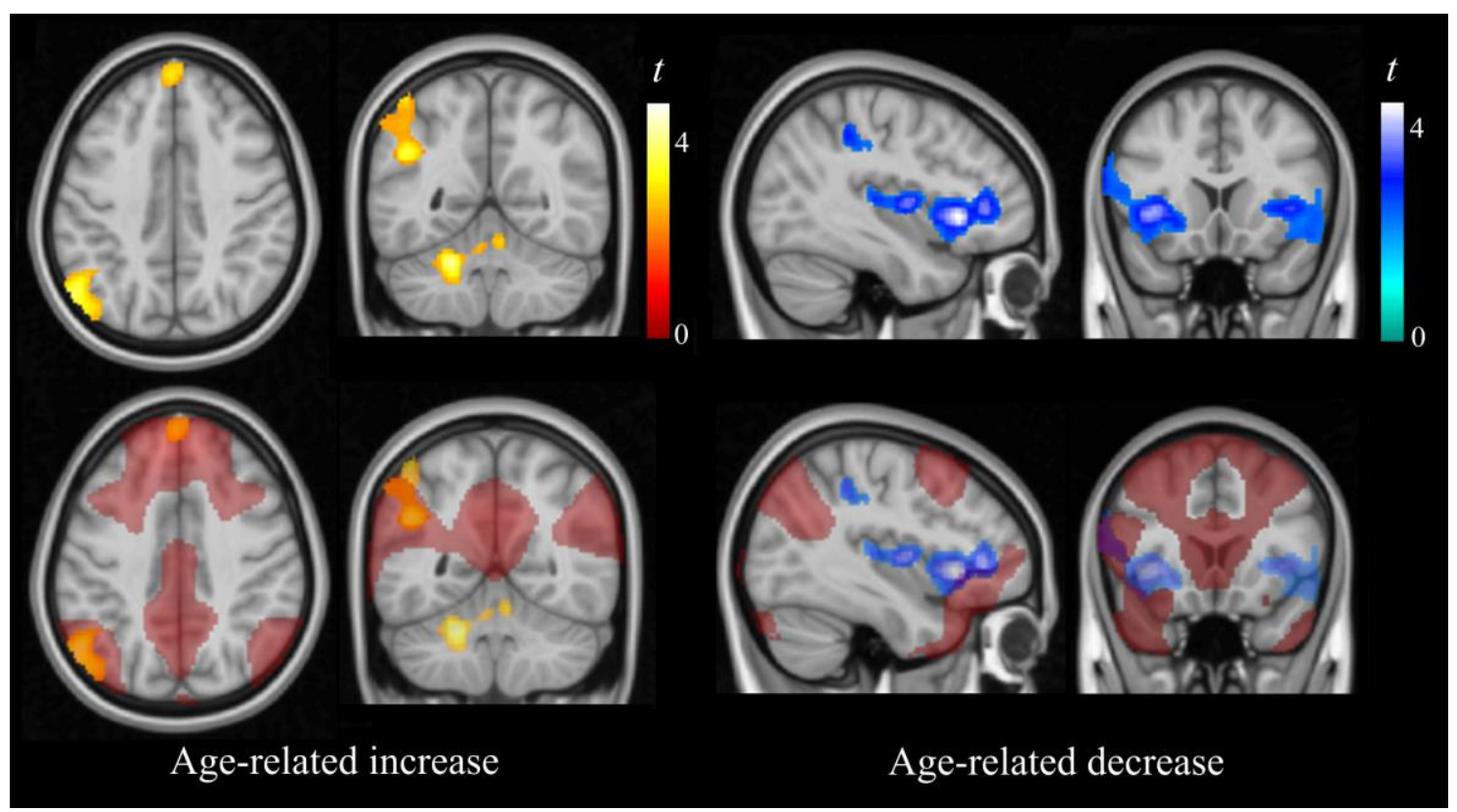

Figure 2. Correlations of age with functional connectivity in the medial frontal seed map. Age was associated with higher functional connectivity between the medial frontal (seed) region and the left angular gyrus, medial frontal cortex and cerebellum (left panels), and with lower functional connectivity between the seed and the lateral frontal cortex/insula region bilaterally (right panels). The right hemisphere corresponds to the right side of axial and coronal views. 


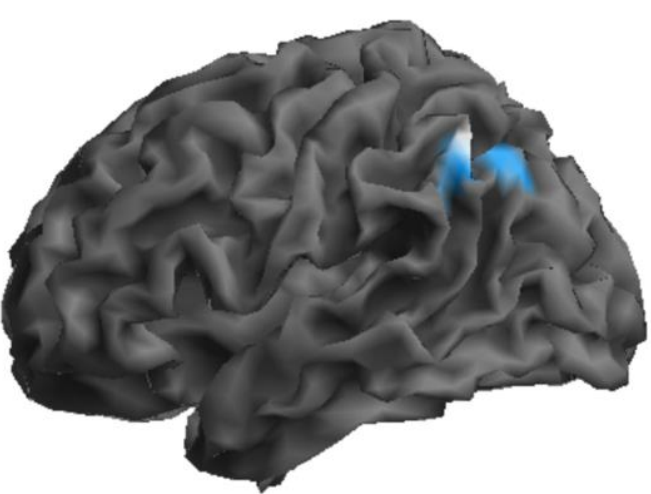

Pollutant-related decrease

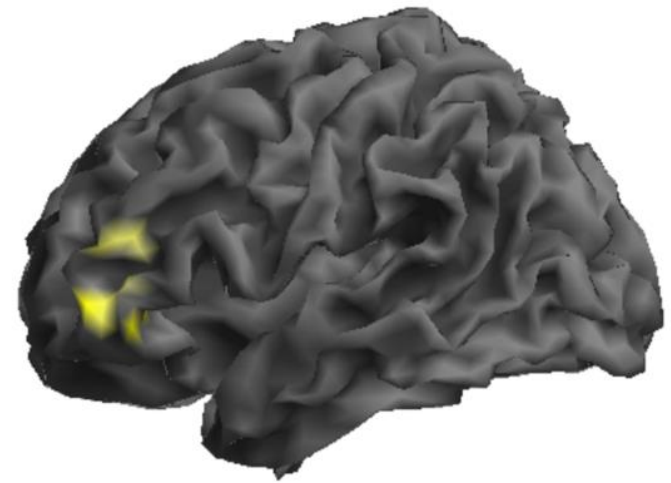

Pollutant-related increase

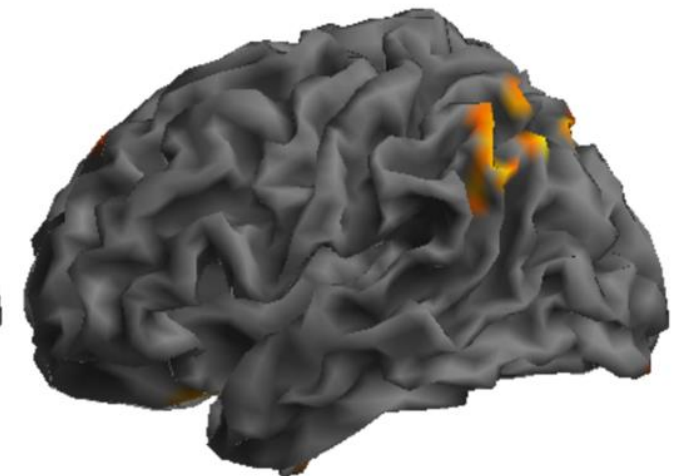

Age-related increase

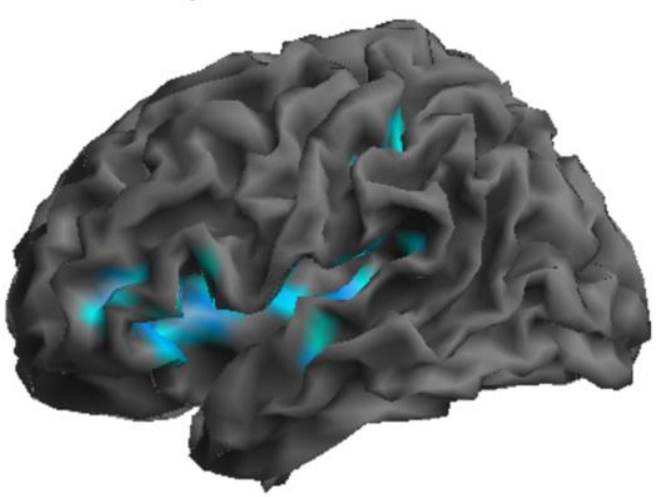

Age-related decrease

Figure 3. 3D rendering display of urban pollution and age effects in the medial frontal seed map. Age and air pollution were associated with opposite effects on functional connectivity in notably similar areas, thus indicating that higher exposure may interfere with the normal development of functional connections. 


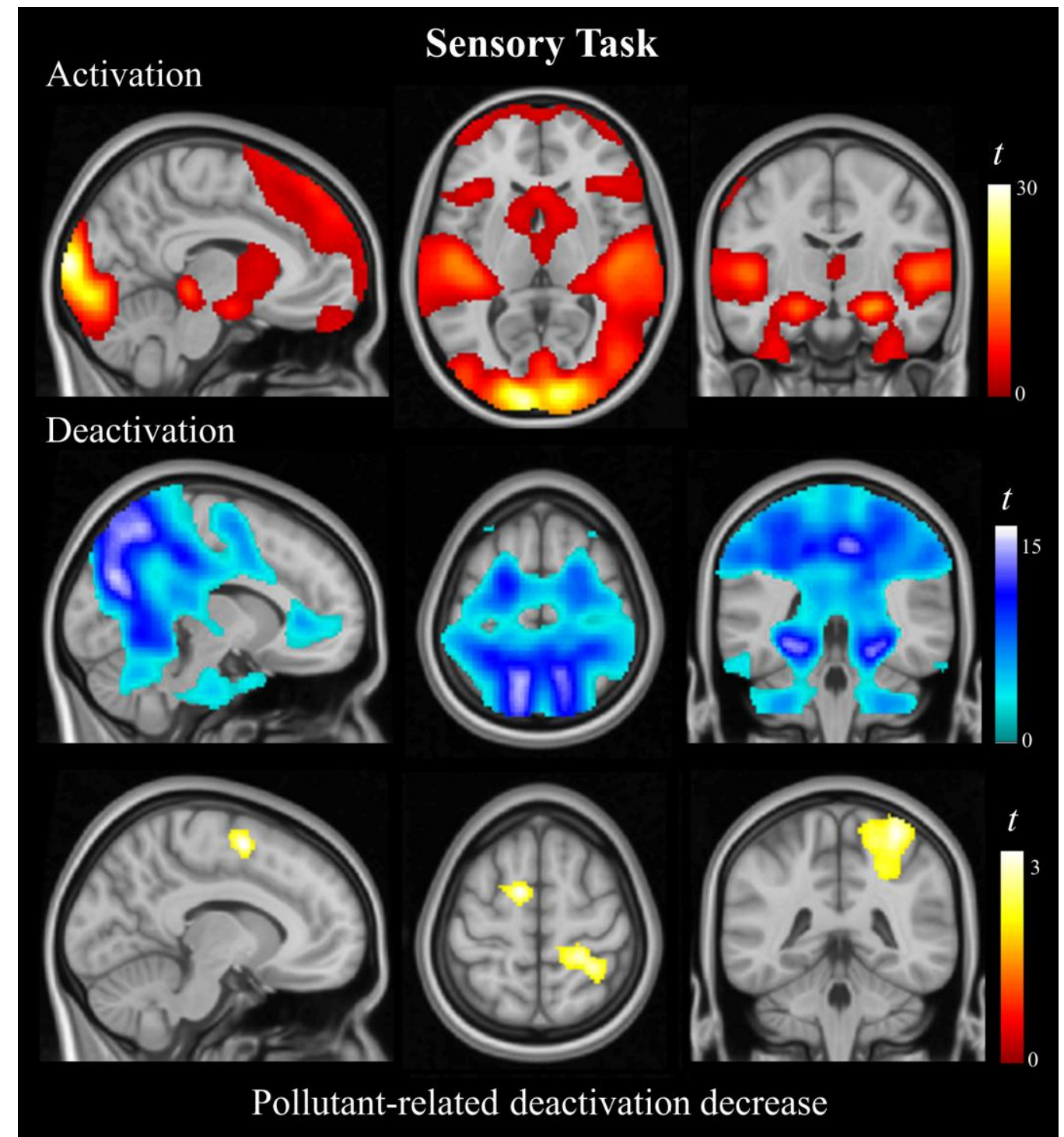

Figure 4. Sensory task results. One-sample group activation (top panel) and deactivation (middle panel) maps showing brain response to passive viewing and listening. Air pollution was associated with lower deactivation in the supplementary motor area and somatosensory cortex included in the deactivation map (bottom panel). The right hemisphere corresponds to the right side of axial and coronal views. 


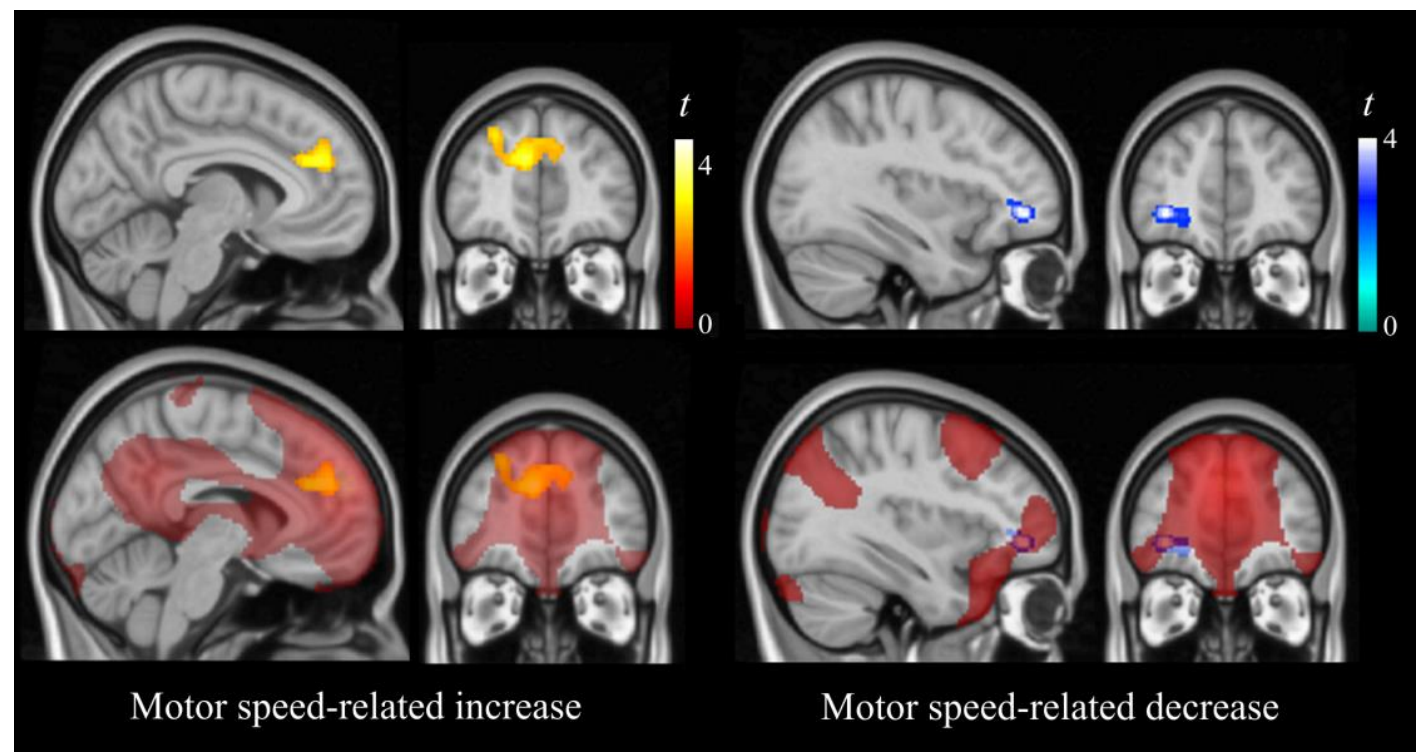

Figure 5. Correlations of performance (motor speed) with functional connectivity in the medial frontal seed map. Faster responses were associated with higher functional connectivity within a core region of the network (medial frontal cortex), and with lower functional connectivity in the lateral boundary of the network. The right hemisphere corresponds to the right side of coronal views. 


\section{Supplementary Material}

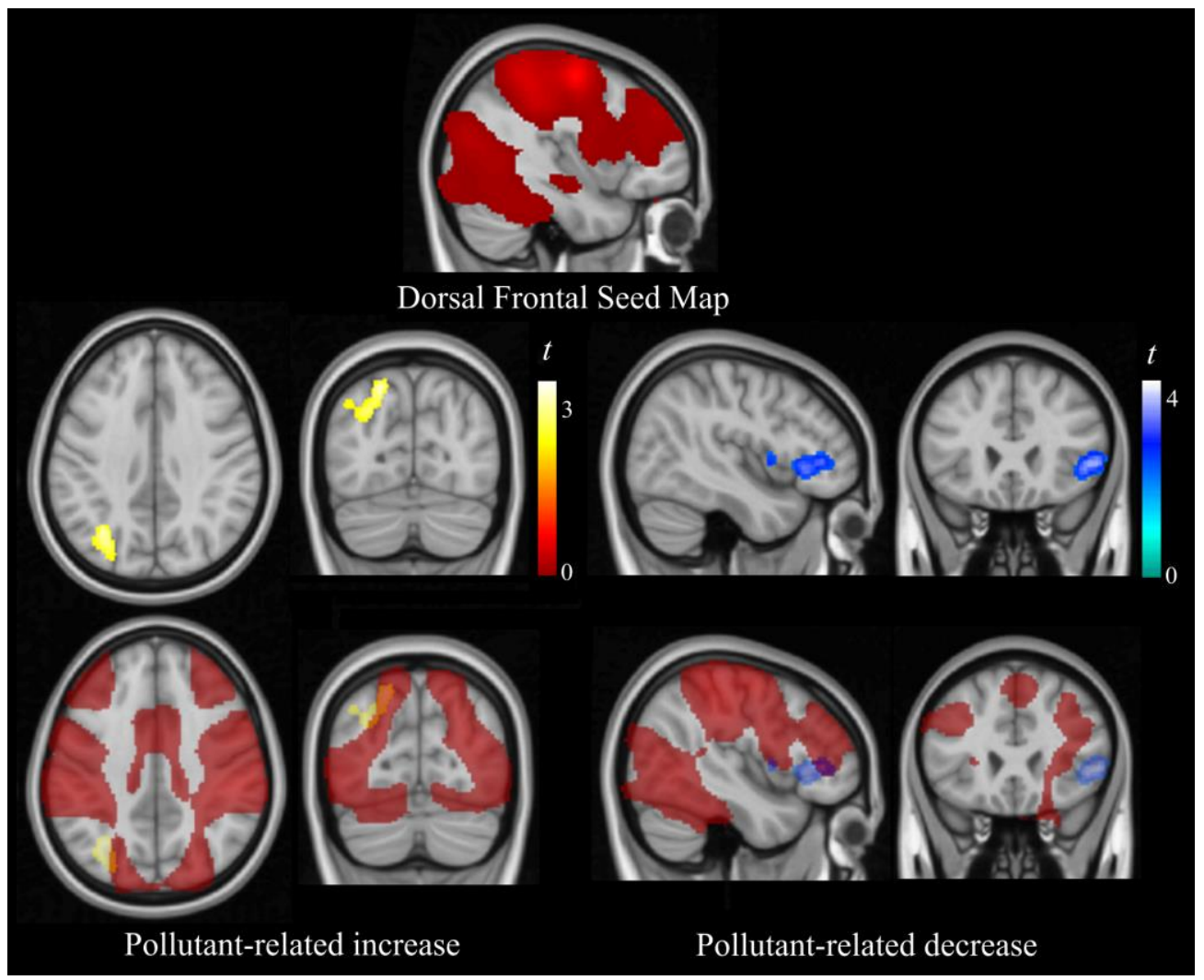

Supplementary Figure 1. Correlations of urban pollution with functional connectivity in the dorsal frontal seed map. Higher pollution levels were associated with higher functional connectivity between the dorsal frontal (seed) region and the parietal cortex (left panels), and lower functional connectivity between the seed and the lateral fontal cortex. Note that these results show the opposite direction to that of the medial frontal analysis (main text Figure 1). The right hemisphere corresponds to the right side of axial and coronal views. 


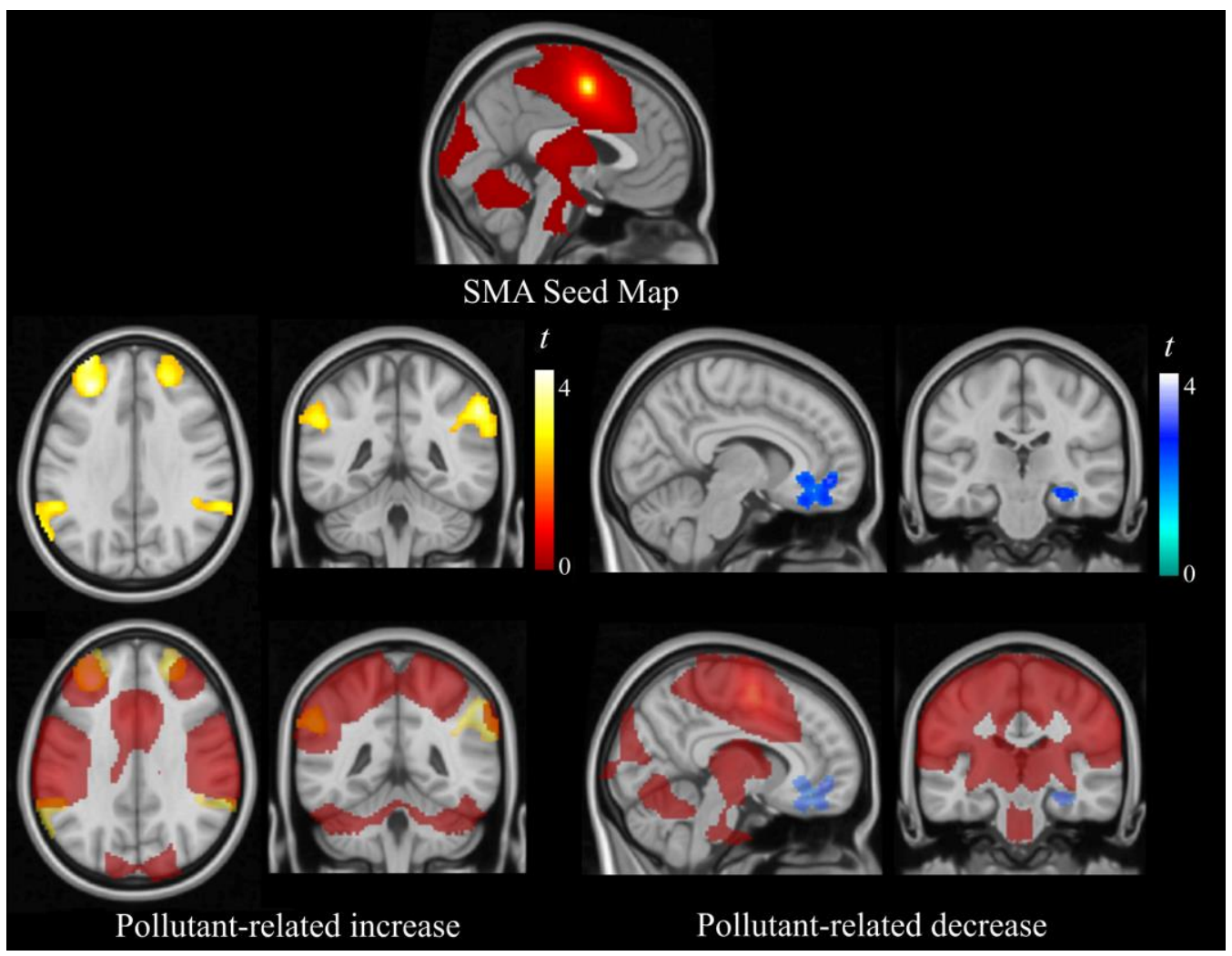

Supplementary Figure 2. Correlations of urban pollution with functional connectivity in the supplementary motor area (SMA) seed map. Higher pollution levels were associated with higher functional connectivity between SMA and both the parietal and anterior prefrontal cortex bilaterally (left panels), and lower functional connectivity between SMA and both the anterior cingulate cortex and hippocampus (left panels). The right hemisphere corresponds to the right side of axial and coronal views. 